\title{
A Novel Demodulation System Based on Continuous Wavelet Transform
}

\author{
Lanting Fang, ${ }^{1}$ Lenan $\mathrm{Wu}^{1}{ }^{1}$ and Yudong $\mathrm{Zhang}^{2}$ \\ ${ }^{1}$ School of Information Science and Engineering, Southeast University, Nanjing 210096, China \\ ${ }^{2}$ School of Computer Science and Technology, Nanjing Normal University, Nanjing 210023, China
}

Correspondence should be addressed to Lanting Fang; 230139356@seu.edu.cn

Received 28 May 2014; Accepted 15 September 2014

Academic Editor: Jyh-Hong Chou

Copyright (C) 2015 Lanting Fang et al. This is an open access article distributed under the Creative Commons Attribution License, which permits unrestricted use, distribution, and reproduction in any medium, provided the original work is properly cited.

Considering the problem of EBPSK signal demodulation, a new approach based on the wavelet scalogram using continuous wavelet transform is proposed. Our system is twofold: an adaptive wavelet construction method that replaces manual selection existing wavelets method and, on the other hand, a nonlinear demodulation system based on image processing and pattern classification is proposed. To evaluate the performance of the adaptive wavelet and compare the performance of the proposed system with the existing systems, a series of comprehensive simulation experiments is conducted under the environment of uniform white noise, colored noise, and additive white Gaussian noise channel, respectively. Simulation results of different wavelets show that the system using adaptive wavelet has lower bit error rate (BER). Moreover, simulation results of several systems show that the BER of the proposed system is the lowest among all systems, such as amplitude detection, integral detection, and some continuous wavelet transform systems (specific scales and times and maximum lines). In a word, the adaptive wavelet construction proposed in this paper yields superior performances compared with the manual selection, and the proposed system has better performances than the existing systems. Index terms are signal demodulation, adaptive wavelet, continuous wavelet transform, and BER.

\section{Introduction}

Spectrum shortage has been a focus of concern in the field of communications. Due to the rapid development in multimedia services such as high definition (HD) video, ultraHD video, and lossless music, radio spectrum is increasingly needed. In order to meet the increasing demand for communication systems, the research on bandwidth efficient modulations is extremely urgent. Recently, many advanced technologies have been proposed in the field of high efficient modulations which own very high data rates and very high spectra efficiency. Consequently, many researchers begin to pay more attention to this breakthrough [1-3]. The extended binary phase shift keying (EBPSK) modulation, as one kind of high efficient modulation, has very high energy efficiency [4]. A special impacting filter (SIF), which can produce high impact at the phase jumping point, constrict bandwidth, and extend channel capacity is applied in the demodulator [5]. Performance can be elevated by using nonlinear demodulation method [6]. Introducing wavelet transform in demodulation system can achieve great results. Wavelet transform is a mathematical process that is closely linked with the world of graphics [7-9]. Image processing methods are always introduced to deal with CWT scalogram, such as feature selection and background subtraction, which can be seen in [10-12]. Meanwhile, wavelet transform is a common method for image processing $[13,14]$. Furthermore, wavelet theory has been widely used to analyze, evaluate, pick, and detect signals [15-18].

This paper has provided a new EBPSK demodulation system based on the continuous wavelet transform (CWT), and the simulation results show that this system has better performance than the existing demodulation systems. Our demodulation system has to be fulfilled by four modules of SIF, CWT, image processing system (IPS), and pattern classification (PC). In this paper, an adaptive wavelet instead of manual selection existing wavelets has been developed. We construct an adaptive wavelet based on the modulation signal without noise and perform image processing on the wavelet scalogram which is the output of CWT. A feature 
template is generated according to the characteristics of the signal under noise free condition, and the feature template is then used for IPS. Only the information retained after image processing can be used to reveal the signal features. After that, we perform the signal detection by using a pattern recognition method. Section 2.1 shows the system model. Section 2.2 shows the modulation signal model. The CWT and the adaptive wavelet construction method are shown in Section 2.3, while in Section 2.4 we will give a brief presentation of image processing and pattern classification. Section 3 presents the simulation results under the conditions of white noise with uniform distribution, colored noise, and additive white Gaussian noise (AWGN). With the proposed system, the performance of adaptive wavelet construction method and manual selection method is compared. Furthermore, the proposed system using the adaptive wavelet is compared with the existing linear and nonlinear systems, respectively.

\section{Theoretical and Mathematical Preliminaries}

2.1. System Model. The system is composed of three modules, that is, modulation, channel, and demodulation. The modulation system creates the modulated signals, and the channel adds noise to it, the demodulation system is fulfilled by four processes of SIF, CWT, IPS, and PC. The detailed description of the method is illustrated in Figure 1, in which the dashed rectangle shows the demodulation system.

The signal of the channel output can be expressed as $w(k)=z(k)+n(k)$ and the SIF output signal $y(k)$ can be expressed as $y(k)=w(k) * h(k)$, where $h(k)$ is the impulse response of SIF, “*” represents convolution operation. $\mathbf{M}(a, b)$ shows the wavelet scalogram, and $\mathbf{C}(a, b)$ is the IPS output.

\subsection{Mathematical Modulation Model of the EBPSK Signal.} EBPSK is a kind of modulation system with high spectrum efficiency, its modulate signal is defined as follows:

$$
\begin{aligned}
& g_{0}(t)=A \sin 2 \pi f_{c} t, \quad 0 \leq t<T, \\
& g_{1}(\mathrm{t})= \begin{cases}B \sin \left(2 \pi f_{c} t+\theta\right), & 0 \leq t<\tau, \quad 0 \leq \theta \leq \pi \\
A \sin 2 \pi f_{c} t, & 0<\tau \leq t<T,\end{cases}
\end{aligned}
$$

where $g_{0}(t)$ and $g_{1}(t)$, respectively, indicate the modulated signals of bits " 0 " and " 1 ." $\theta$ is the modulating angle. $T=$ $N / f_{c}$ indicates the temporal length of a code, and parameter $f_{c}$ represents the carrier frequency and $N$ the number of carriers. The phase modulation temporal length $\tau=K / f_{c}$ in the bit " 1 " lasted $K \leq N$ cycles of carrier. The amplitude of $g_{1}(t)$ after jumping is equal to that of $g_{0}(t)$, which is represented by $A$. $B$ represents the amplitude of $g_{1}(t)$ before jumping.

\subsection{CWT. The CWT of a signal $f(t)$ is given by}

$$
\begin{aligned}
\mathbf{W}_{f}(a, b) & =\left\langle f, \Psi_{a, b}\right\rangle \\
& =|a|^{-1 / 2} \int_{\mathbb{R}} f(t) \overline{\Psi\left(\frac{t-b}{a}\right)} \mathrm{d} t,
\end{aligned}
$$

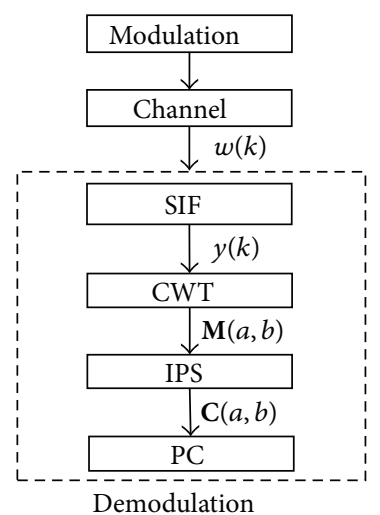

Figure 1: The block diagram of system model.

where " $a$ " represents the scale factor and " $b$ " is the shift factor, and $\Psi_{a, b}(t)=\Psi((t-b) / a)$ is a wavelet function with zero mean as follows:

$$
\int_{-\infty}^{\infty} \Psi(t) \mathrm{d} t=0
$$

CWT is the calculation of the cross covariance between the signal and the wavelet function, which is shifted in time and stretched in scale. The coefficient $\mathbf{W}_{f}(a, b)$ indicates the similarity between the wavelet function and the signal. A larger value of $\mathbf{W}_{f}(a, b)$ indicates a better waveforms match. Wavelet coefficient depends on the wavelet function. Consequently, in order to detect certain signal, the waveform of the wavelet function similar to the signal should be chosen. The advantage of the CWT over the classical template matching methods arises from the special properties of the wavelet template, allowing optimal scale separation of the signal [19].

It is essential to choose the mother wavelet to make the easiest identification of the wavelet scalogram feature. The existing wavelet functions are divided into five main types: finite impulse response (FIR) filter wavelet, such as Haar, Daubechies (db), Coiflets (coif), and Symlets (sym); biorthogonal wavelet with a FIR filter, such as Bior Splines (bior); filter without FIR, but with a scale equation, such as Meyer (meyr) wavelet; wavelet without FIR filter or scale equation, such as Morlet (morl) and Mexican hat (mexh); complex wavelet with a finite impulse and a scale equation, such as complex Gaussian and Shannon.

The selection of a particular wavelet function depends on the scalogram features to be extracted $[20,21]$. As the adaptive wavelet waveform can be adjusted as requested, using the adaptive wavelet can achieve superior results than manual selection existing wavelets. We transform bit "1" signal under the noiseless condition though SIF, and then we customize the SIF output to function $\Psi_{\text {EBPSK }}$ by orthogonal projection for constants. As most of the information is concentrated at the beginning of a bit, we cut out $1 / 2$ temporal length of a bit in order to fit custom wavelet, which starts from $3 T / 4$ of the prior code. The adaptive wavelet waveform is shown in Figure 2. 


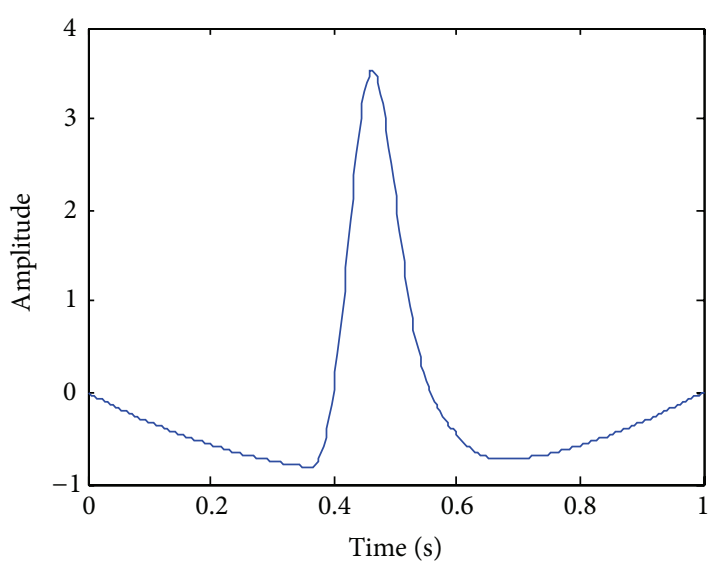

FIGURE 2: Adaptive wavelet.

2.4. IPS and PC. The wavelet scalogram of coefficients $\mathbf{M}$ generated by the signal $y(k)$ is given by the following equation:

$$
\begin{aligned}
\mathbf{M}(a, b) & =\left\langle y, \Psi_{\mathrm{EBPSK}}\right\rangle \\
& =|a|^{-1 / 2} \int_{\mathbb{R}} y(k) \overline{\Psi_{\mathrm{EBPSK}}\left(\frac{k-b}{a}\right)} \mathrm{d} k,
\end{aligned}
$$

where $\Psi_{\text {EBPSK }}$ is the custom wavelet function.

The wavelet scalogram of coefficients $\mathbf{M}$ includes the characteristic information as well as the redundant information. As scalogram features are focused in fixed region, a wavelet scalogram windowing was also proposed for extracting feature information. Matrix $\mathbf{C}$ transformed by the IPS can be expressed as follows:

$$
\mathbf{C}(a, b)=\mathbf{M}(a, b) \times \mathbf{I}(a, b),
$$

where matrix I is the window matrix, and it uses " 1 " and " 0 " to indicate the highlight regions and the background regions of bit " 1 ".

We transform a bit "1" signal without noise through SIF and CWT, while $\mathbf{M}^{\prime}$ represents the CWT output. Matrix I can be expressed as follows:

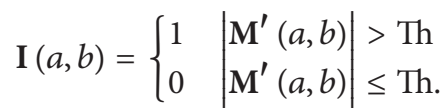

Th is a threshold used to distinguish the highlight regions and the background regions, and it is determined by the signal and the environment. In Section 3, we use Th $=0$, which indicates that all nonzero sections are highlight sections.

Figure 3(a) shows the output of a noise-free signal through SIF. While Figure 3(b) shows the wavelet scalogram, it is displayed with "pink" colormap using the maximum absolute value in all scales. The coefficients line for scale $a=$ 40 is demonstrated in Figure 3(c).

Pattern classification is the kernel of image classification, and we utilized the methods of this field in signal classification to achieve the desired results.
The Euclidean distance between transformed matrix $\mathbf{C}$, which is the output of IPS, and the wavelet scalogram template $\mathbf{W}_{K}(K=1,2)$, is given by the following equation:

$$
D_{K}=\left\|\mathbf{C}-\mathbf{W}_{K}\right\|_{F}
$$

where $\mathbf{W}_{1}$ represents the scalogram template of bit "1" signal and $\mathbf{W}_{0}$ represents that of bit " 0 " signal.

$\mathbf{M}^{\prime \prime}$ represents the CWT output of noiseless bit " 0 ." Coefficient templates $\mathbf{W}_{1}$ and $\mathbf{W}_{0}$ are, respectively, defined by the following equations:

$$
\begin{aligned}
& \mathbf{W}_{1}(a, b)=\mathbf{M}^{\prime}(a, b) \times \mathbf{I}(a, b), \\
& \mathbf{W}_{0}(a, b)=\mathbf{M}^{\prime \prime}(a, b) \times \mathbf{I}(a, b) .
\end{aligned}
$$

Since the distribution of data for component of each dimension is not the same, we have improved formula (7) by standardizing each component to equal mean and variance.

$\overline{\mathbf{X}}(a, b)$ is the mean value of each sample set $(\mathbf{C}(a, b)$, $\left.\mathbf{W}_{1}(a, b), \mathbf{W}_{2}(a, b)\right)$, and the standard deviation of each sample set $\mathbf{S}(a, b)$ can be expressed as follows:

$$
\begin{aligned}
\mathbf{S}^{2}(a, b)= & \frac{(\mathbf{C}(a, b)-\overline{\mathbf{X}}(a, b))^{2}}{3} \\
& +\frac{\left(\mathbf{W}_{1}(a, b)-\overline{\mathbf{X}}(a, b)\right)^{2}}{3} \\
& +\frac{\left(\mathbf{W}_{0}(a, b)-\overline{\mathbf{X}}(a, b)\right)^{2}}{3} .
\end{aligned}
$$

After improving, we can define the Euclidean distance " $D_{1}$ " as follows:

$$
D_{1}=\left\|\frac{\mathbf{C}-\mathbf{W}_{1}}{\mathbf{S}}\right\|_{F} .
$$

The improved Euclidean distance " $D_{0}$ " is defined as follows:

$$
D_{0}=\left\|\frac{\mathbf{C}-\mathbf{W}_{0}}{\mathbf{S}}\right\|_{F} .
$$

The signal characteristics can be detected by Euclidean distance, and we determine that the signal is bit " 1 " if $D_{0}>D_{1}$; otherwise, we will consider the signal as bit " 0 ."

The simulation under the AWGN condition is shown in Figure 4. Figure 4(a) shows the SIF output of signal mixed with noise. The wavelet scalogram $\mathbf{M}$ and the processed matrix $\mathrm{C}$ are shown in Figures 4(b) and 4(c), respectively, where we identified the figures with "pink" colormap using the maximum absolute value in all scales.

\section{Experiments and Results}

In order to validate the proposed methodology of the CWT based demodulation system, introduced in Section 2, we compared it with several existing methods (ID, AD, ML, SST, and the proposed system adopting manual selection wavelet). 


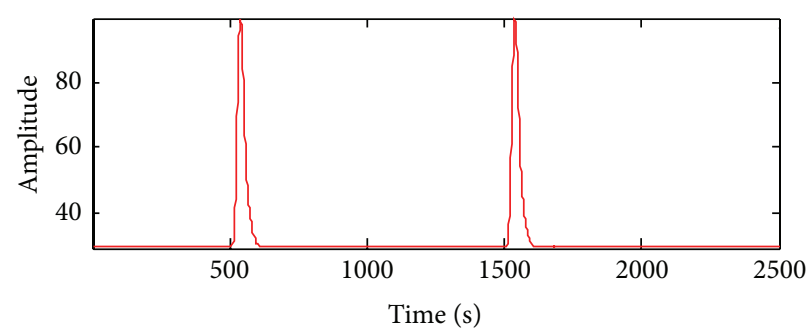

(a)

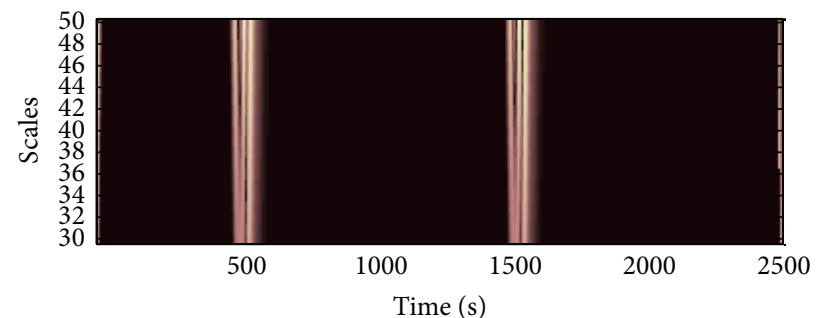

(b)

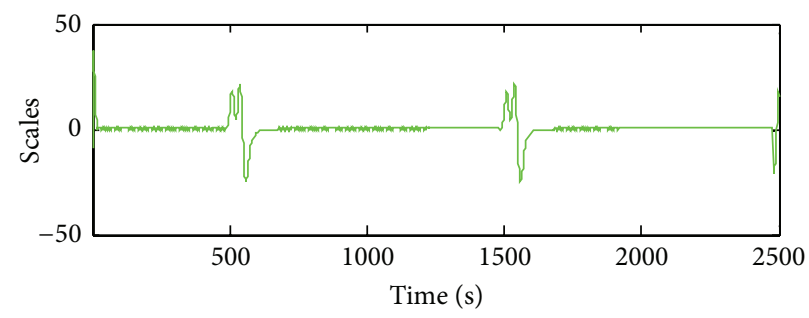

(c)

FIGURE 3: Simulation under noise free condition: (a) SIF output, (b) wavelet scalogram, and (c) coefficients line for scale $a=40$.

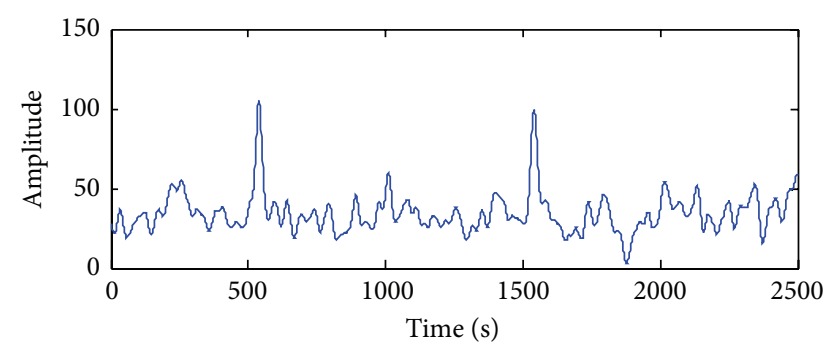

(a)

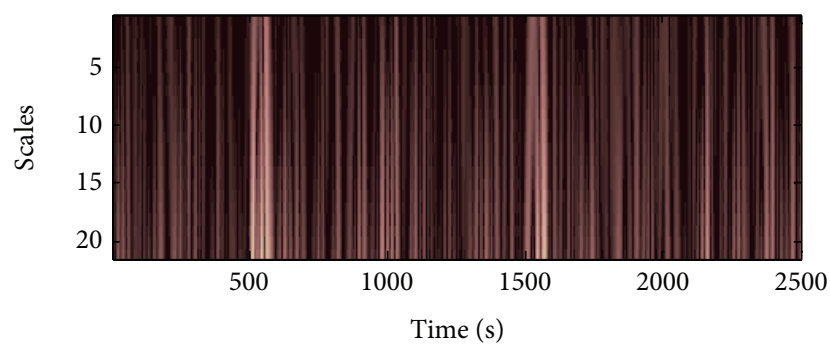

(b)

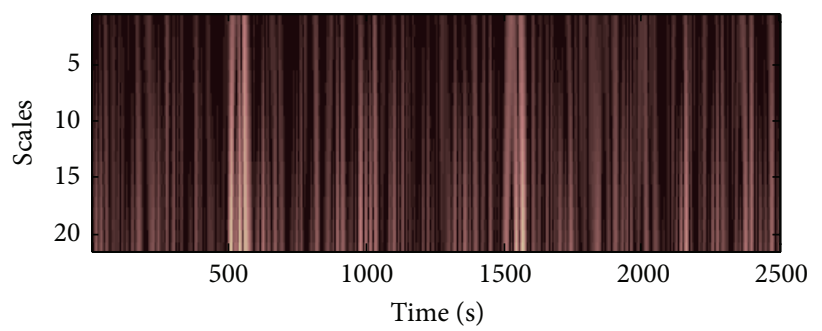

(c)

FIGURE 4: Simulation under AWGN condition: (a) SIF output, (b) wavelet scalogram, and (c) IPS output.

The simulations assumed that the system has 1000 symbols for training and the reported bit error rate (BER) is computed using $1.5 \times 10^{5}$ symbols. $K=2,8,16$ indicate that the simulation model is binary, octal, and hexadecimal. The carrier frequency $f_{c}$ and parameter $N$ can impact the power spectral density of the EBPSK signal. Amplitude $A$ determines the signal strength of bit " 0 " and jumped bit " 1 " and $B$ the signal strength of bit "1" before jumping.

In this paper, we only discuss the case of $K=2$, and we choose the carrier frequency $f_{c}=30 \mathrm{MHZ}$ and parameters $N=50, A=B=1$, and $\theta=\pi$. The experiments are sampled at the rate of $f_{s}=300 \mathrm{MHZ}$.
3.1. Performance Comparisons of the System Proposed and Several Well-Known Systems. We compared the performance of the proposed system adopting adaptive wavelet with the nonlinear systems (ID and AD) under different kinds of environments, such as white noise with uniform distribution, AWGN, and colored noise.

Figure 5(a) depicts the simulation result under the condition of white noise with uniform distribution. It can be seen in Figure 5(a) that while SNR $<-3 \mathrm{~dB}$ BER of the proposed system is similar to that of $\mathrm{AD}$ and ID. While using SNR > $-3 \mathrm{~dB}$, our method has a lower BER compared to the wellknown $\mathrm{AD}$ and ID. 


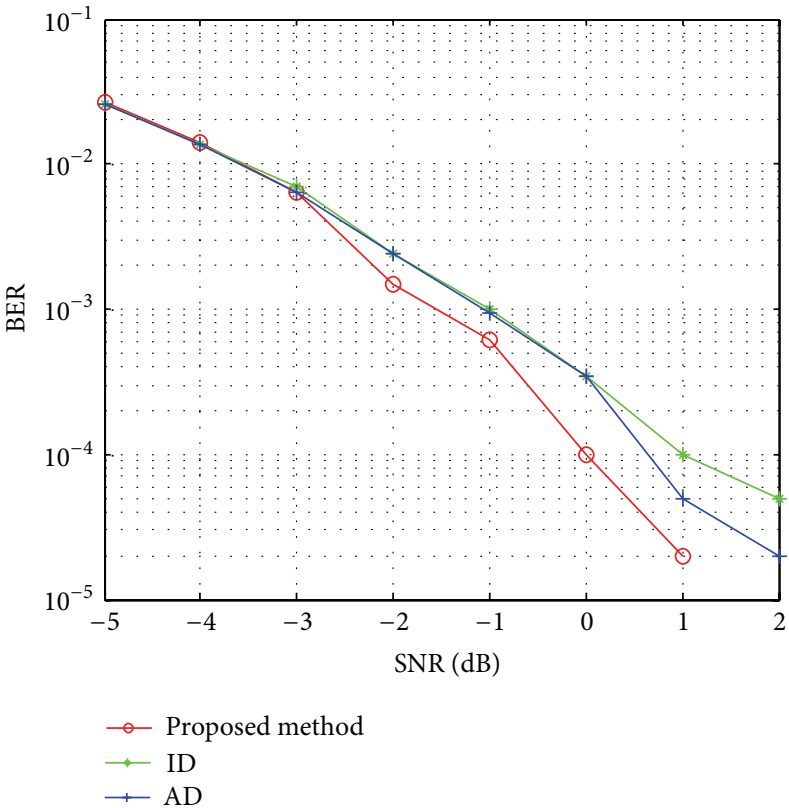

(a)

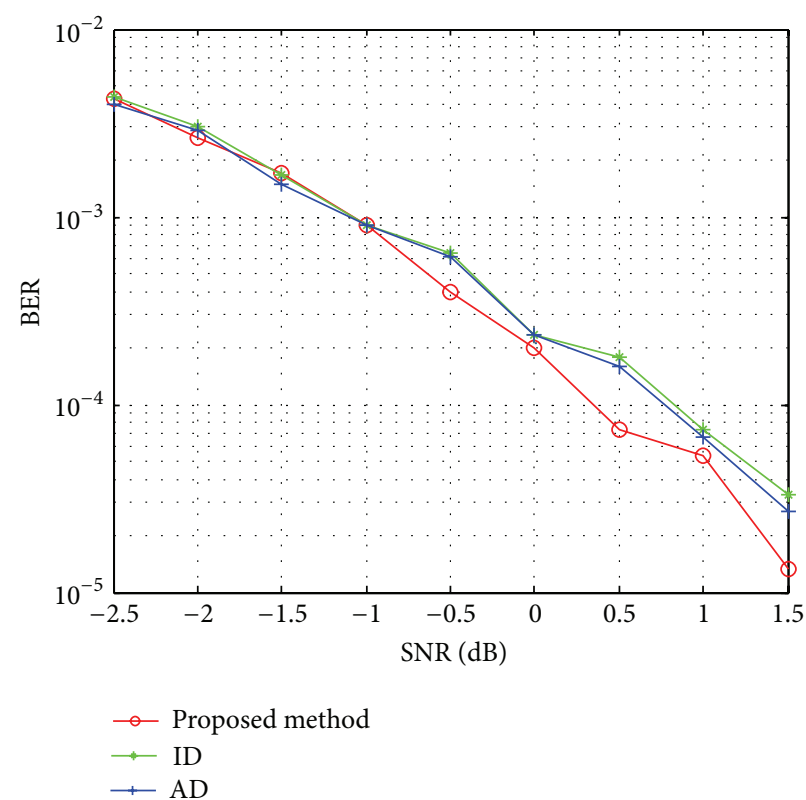

(b)

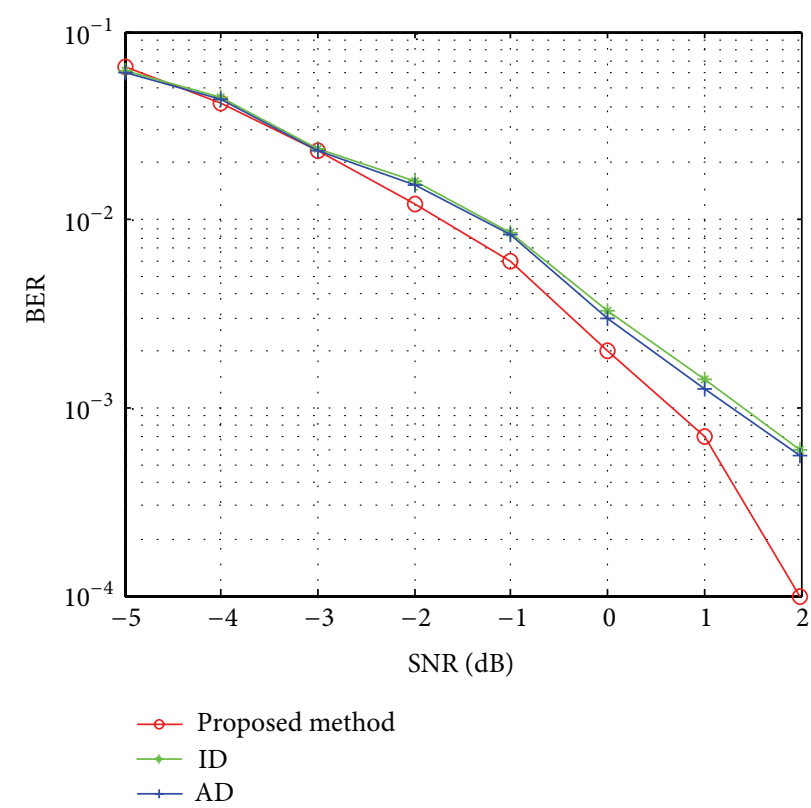

(c)

FIGURE 5: ID, AD, and the proposed method under different kinds of environments: (a) white noise with uniform distribution, (b) AWGN, and (c) colored noise.

Figure 5(b) shows the simulation results under the environment of additive white Gaussian noise (AWGN). While SNR $<-1 \mathrm{~dB}$, BER of the proposed system has no advantage; however, when the SNR $>-1 \mathrm{~dB}$, our system has a better result.

Figure 5(c) demonstrates the excellent performance of the proposed method under the environment of colored noise. Actually, in communications, the real channel is always bandwidth limited, which results in colored noise or band-limited noise. We have generated the colored noise by low-pass filtering the Gaussian white noise. The parameters of this low-pass filter are pass-band corner frequency $W_{p}=0.6$, stop-band corner frequency $W_{s}=1$, pass-band ripple in decibels $R_{p}=0.5$, and stop-band attenuation $R_{s}=40 \mathrm{~dB}$.

3.2. Experimental Results of Systems Based on CWT. To show the competitive performance of our system, some experiment results of other CWT demodulation systems such as specific scales and times (SST) and maximum lines (ML) are shown in Figure 6. All CWT demodulation systems were performed 


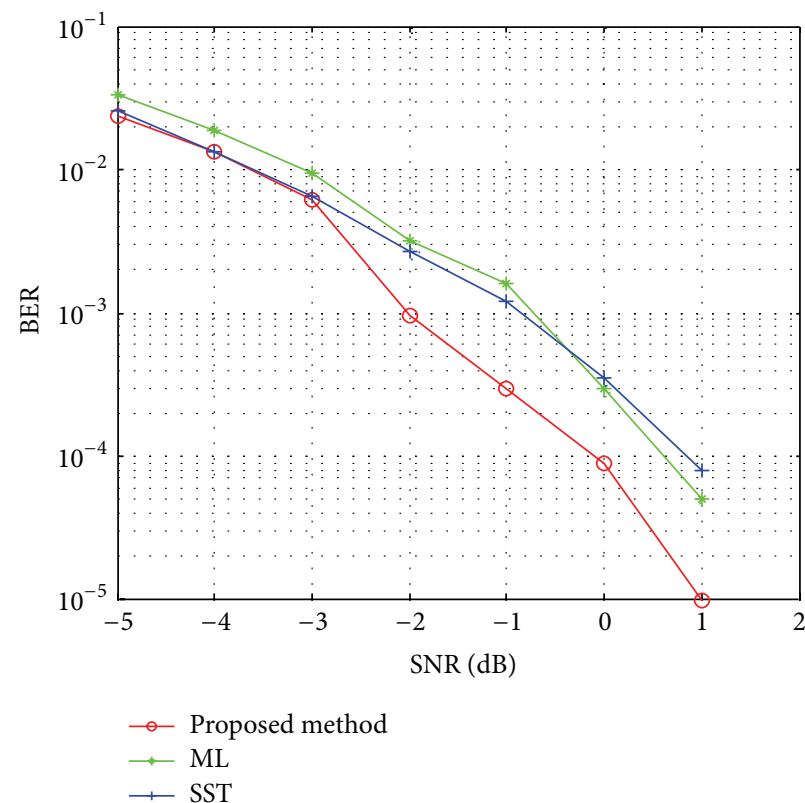

(a)

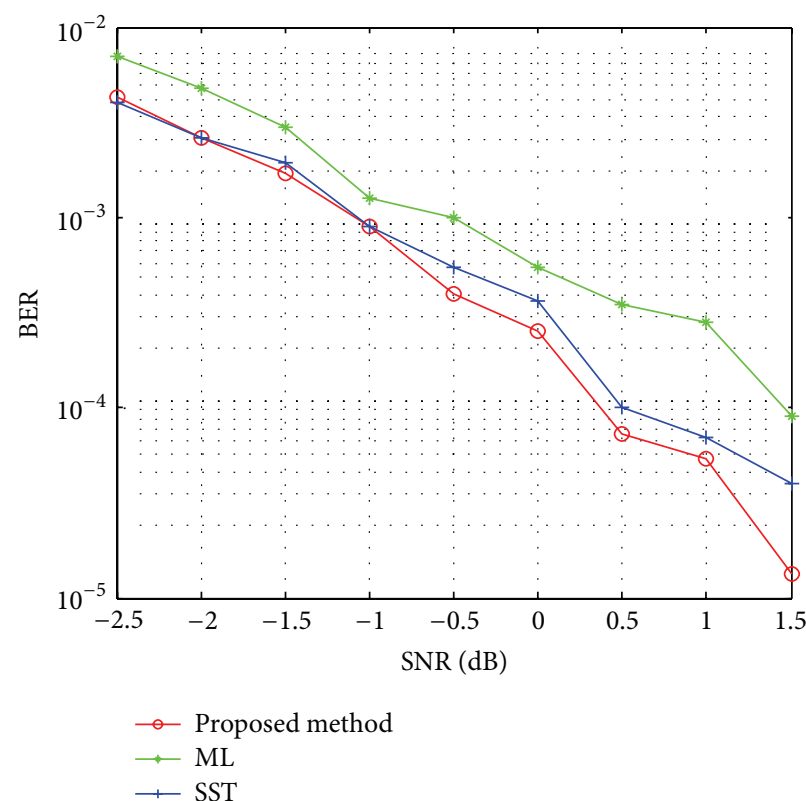

(b)

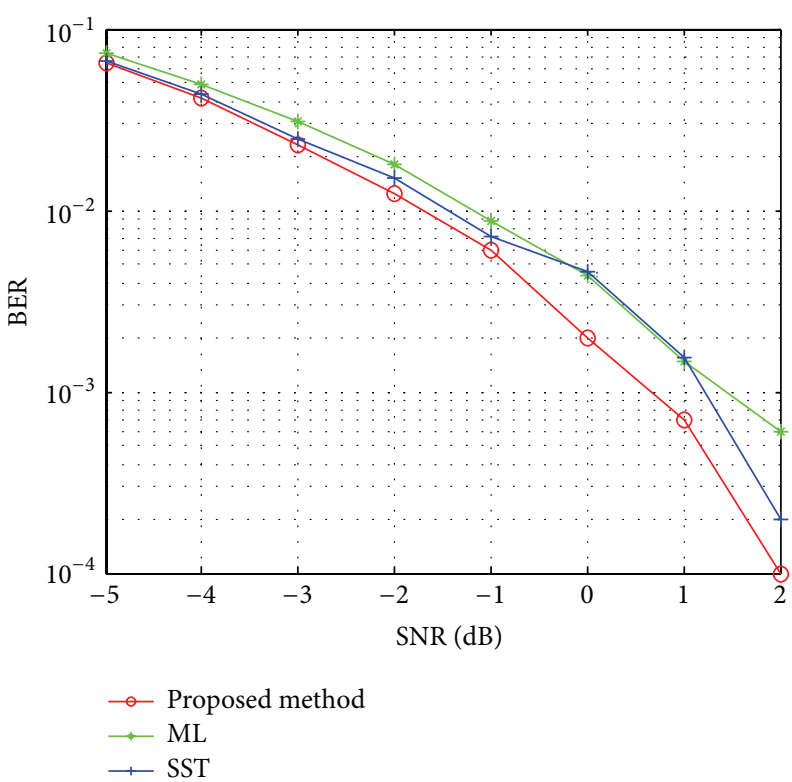

(c)

FIGURE 6: ML, SST, and the proposed method under different kinds of environments: (a) white noise with uniform distribution, (b) AWGN, and (c) colored noise.

using adaptive wavelet. As Figure 6 reveals, ML algorithm has the highest BER under the environment of AWGN. ML and SST have a similar performance under the condition of white noise with uniform distribution and colored noise. While the proposed method yields the lowest BER under all studied environments.

3.3. Simulation Results of Different Wavelets. In order to demonstrate the superiority of the adaptive wavelet, we simulated the proposed system adopting manual selection existing wavelets which include db2, morl, Meyer, mexh, and sym2. The demodulation performances are presented in Figure 7, for all the studied noise types.

Figure 7(a) shows the simulation results of the adaptive wavelet and several existing wavelets under the condition of white noise with uniform distribution. Experiments under the condition of AWGN are shown in Figure 7(b). Figure 7(c) shows the simulation results under the condition of colored 


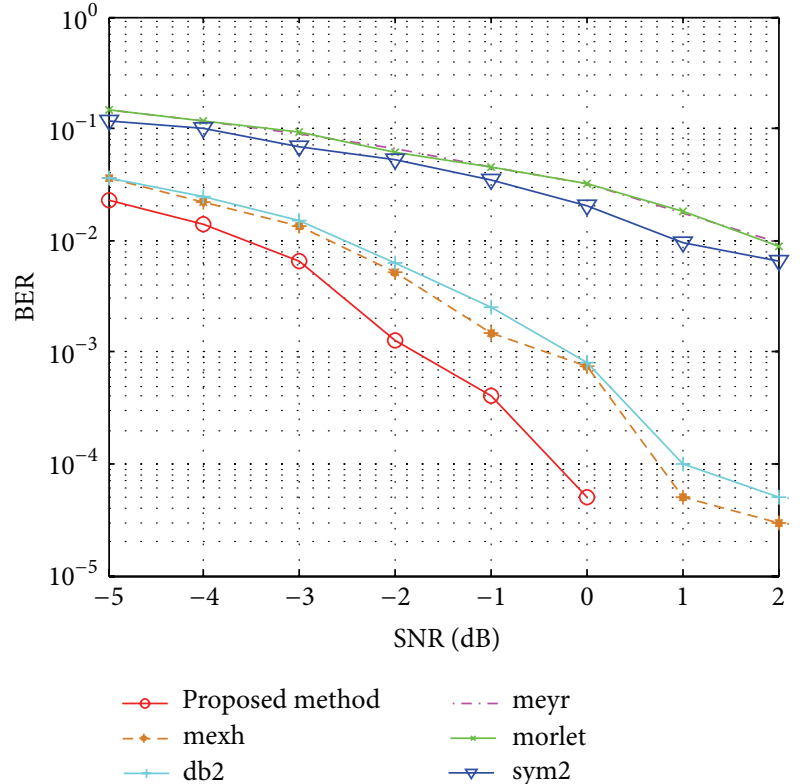

(a)

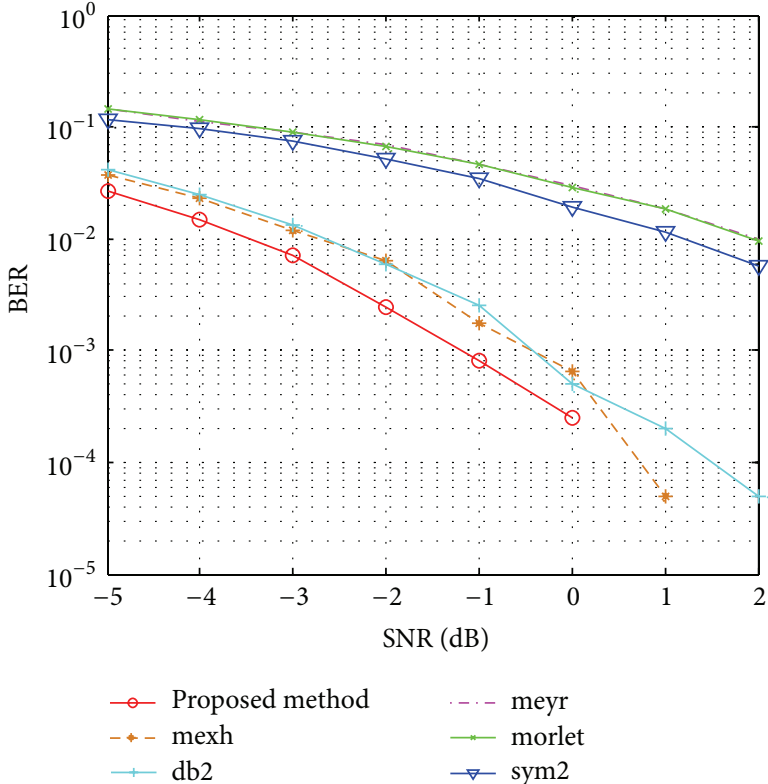

(b)

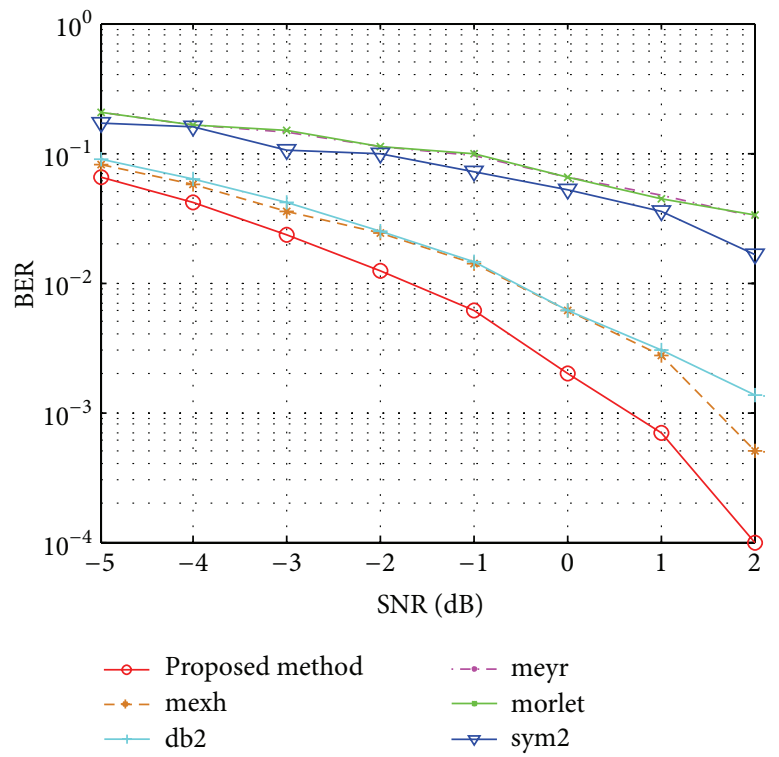

(c)

FIGURE 7: Proposed system adopting different wavelets under studied environments: (a) white noise with uniform distribution, (b) AWGN, and (c) colored noise.

noise. It can be realized from Figure 7 that mexh and $\mathrm{db} 2$ are more suitable to EBPSK compared with the other existing wavelets; however, the adaptive wavelet yields the best performance in all wavelets.

The computational complexity of all studied systems is shown in Table $1 . n$ is the length of code, from which we see that ID and AD's asymptotical complexity is proportional with the parameter $n$ and the complexity of ML, SST, and the proposed system using manual selection wavelets is the same as the complexity of the proposed system using adaptive wavelet. They are all quadratic-time complexity. Although $\mathrm{ID}$ and $\mathrm{AD}$ have lower complexity, the proposed system has better demodulation performance than them, and their BER are higher than the proposed method, which can be seen in Figure 5. ML, SST, and the proposed system adopting manual selection wavelets have the same complexity as the proposed method, while their BER are higher than the proposed method, which can be seen in Figures 6 and 7 .

\section{Discussion}

This paper presents an adaptive wavelet construction method. Unlike manual wavelet selection methods, this algorithm 
TABLE 1: Computational complexity.

\begin{tabular}{lc}
\hline Methods & Complexity \\
\hline ID & $O(n)$ \\
AD & $O(n)$ \\
ML & $O\left(n^{2}\right)$ \\
SST & $O\left(n^{2}\right)$ \\
Our system & \\
$\quad$ Adaptive wavelet & $O\left(n^{2}\right)$ \\
$\quad$ Manual selection & $O\left(n^{2}\right)$ \\
\hline
\end{tabular}

does not need to select wavelet in the light of experiments. The wavelet is constructed based on the transformed signal waveform. Therefore, the adaptive construction method has better real-time capability and higher accuracy.

SIF, CWT, IPS, and PC are introduced into the demodulation system. CWT transforms the signal to the wavelet scalogram. IPS highlights the signal information and enhances the distinction between the signal and the noise. PC fulfills the signal detection, and the detection results show that the proposed system obtains lower BER than the existing systems.

The proposed approach can be combined with any signal classification and detection system. The wavelet scalogram classification integrated with the image processing system can be used in fault detection, medical signal processing, and particular signal picking systems.

Our demodulation system has no advantage in terms of complexity, and the system may reflect the limited advantage at extremely low SNR, probably because image processing procedure enhances the noise in a manner similar to the signal information at extremely low SNR. For further improvement in the demodulation performance and more reduction in the complexity of the algorithm, future work will focus on the feature extraction and the system optimization.

\section{Conclusion}

A novel EBPSK demodulation system based on CWT is proposed in this study. An adaptive wavelet is proposed to transform the SIF output signal. IPS is utilized to enhance the distinction between the signal and the noise. The system is detected by PC. The performance of the proposed system is checked under the condition of white noise with uniform distribution, AWGN, and colored noise. The results are compared with $\mathrm{AD}, \mathrm{ID}, \mathrm{ML}$, and SST techniques. Our results show that the proposed system has lower BER. The proposed system adopting adaptive wavelet is compared with the proposed system adopting traditional manual selection wavelets, and the results show that the adaptive wavelet is more suitable to the transformed signal.

\section{Conflict of Interests}

The authors declare that there is no conflict of interests regarding the publication of this paper.

\section{Acknowledgments}

The work is supported by the National Key Technology R\&D Program under the Grant 2012BAH15B00 and the National Natural Science Foundation of China (nos. 61271204 and 610011024).

\section{References}

[1] J. Zhang, W. Bai, L. Cai, Y. Xu, G. Song, and Q. Gan, "Observation of ultra-narrow band plasmon induced transparency based on large-area hybrid plasmon-waveguide systems," Applied Physics Letters, vol. 99, no. 18, Article ID 181120, pp. 1-3, 2011.

[2] X. Liu, "A novel ultra-narrow transmission-band fiber Bragg grating and its application in a single-longitudinal-mode fiber laser with improved efficiency," Optics Communications, vol. 280, no. 1, pp. 147-152, 2007.

[3] C. Moser and F. Havermeyer, "Ultra-narrow-band tunable laserline notch filter," Applied Physics B, vol. 95, no. 3, pp. 597601, 2009.

[4] L. Wu and M. Feng, "On BER performance of EBPSK-MODEM in AWGN channel," Sensors, vol. 10, no. 4, pp. 3824-3834, 2010.

[5] M. Feng, L. Wu, J. Ding, and C. Qi, "BER analysis and verification of EBPSK system in AWGN channe," IEICE Transactions on Communications, vol. E94-B, no. 3, pp. 806-809, 2011.

[6] X. Chen and L. Wu, "Nonlinear demodulation and channel coding in EBPSK scheme," The Scientific World Journal, vol. 2012, Article ID 180469, 7 pages, 2012.

[7] Y. Zhang, S. Wang, Y. Huo, L. Wu, and A. Liu, "Feature extraction of brain MRI by stationary wavelet transform and its applications," Journal of Biological Systems, vol. 18, no. 1, pp. 115$132,2010$.

[8] T. Cheng, B. Rivard, A. G. Sánchez-Azofeifa, J.-B. Féret, S. Jacquemoud, and S. L. Ustin, "Predicting leaf gravimetric water content from foliar reflectance across a range of plant species using continuous wavelet analysis," Journal of Plant Physiology, vol. 169, no. 12, pp. 1134-1142, 2012.

[9] Y. Zhang, S. Wang, G. Ji, and Z. Dong, "Genetic pattern search and its application to brain image classification," Mathematical Problems in Engineering, vol. 2013, Article ID 580876, 8 pages, 2013.

[10] T.-P. Le and P. Paultre, "Modal identification based on continuous wavelet transform and ambient excitation tests," Journal of Sound and Vibration, vol. 331, no. 9, pp. 2023-2037, 2012.

[11] X. Jiang, Z. J. Ma, and W.-X. Ren, "Crack detection from the slope of the mode shape using complex continuous wavelet transform," Computer-Aided Civil and Infrastructure Engineering, vol. 27, no. 3, pp. 187-201, 2012.

[12] A. Lazaro, A. Ramos, D. Girbau, and R. Villarino, "Chipless UWB RFID tag detection using continuous wavelet transform," IEEE Antennas and Wireless Propagation Letters, vol. 10, pp. 520-523, 2011.

[13] Y. Zhang, S. Wang, and G. Ji, "A rule-based model for bankruptcy prediction based on an improved genetic ant colony algorithm," Mathematical Problems in Engineering, vol. 2013, Article ID 753251, 10 pages, 2013.

[14] Y. Zhang, S. Wang, G. Ji, and Z. Dong, "An MR brain images classifier system via particle swarm optimization and kernel support vector machine," The Scientific World Journal, vol. 2013, Article ID 130134, 9 pages, 2013. 
[15] J. T. Bialasiewicz, D. Gonzalez, J. Balcells, and J. Gago, "Waveletbased approach to evaluation of signal integrity," IEEE Transactions on Industrial Electronics, vol. 60, no. 10, pp. 4590-4598, 2013.

[16] S. Banerjee and M. Mitra, "Application of cross wavelet transform for ECG pattern analysis and classi?cation," IEEE Transactions on Instrumentation and Measurement, vol. 63, no. 2, pp. 326-333, 2014.

[17] N. Karamzadeh, G. J. Doloei, and A. M. Reza, "Automatic earthquake signal onset picking based on the continuous wavelet transform," IEEE Transactions on Geoscience and Remote Sensing, vol. 51, no. 5, pp. 2666-2674, 2013.

[18] F. B. Costa, "Fault-induced transient detection based on realtime analysis of the wavelet coefficient energy," IEEE Transactions on Power Delivery, vol. 29, no. 1, pp. 140-153, 2014.

[19] V. Bostanov, "BCI competition 2003-data sets Ib and IIb: feature extraction from event-related brain potentials with the continuous wavelet transform and the t-value scalogram," IEEE Transactions on Biomedical Engineering, vol. 51, no. 6, pp. 10571061, 2004.

[20] L. Smital, M. Vitek, J. Kozumplik, and I. Provaznik, "Adaptive wavelet wiener filtering of ECG signals," IEEE Transactions on Biomedical Engineering, vol. 60, no. 2, pp. 437-445, 2013.

[21] J. Gilles, "Empirical wavelet transform," IEEE Transactions on Signal Processing, vol. 61, no. 16, pp. 3999-4010, 2013. 


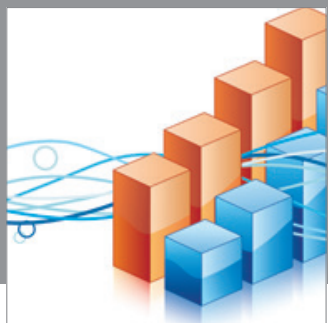

Advances in

Operations Research

mansans

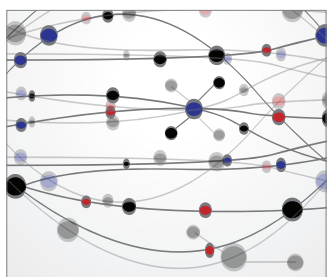

The Scientific World Journal
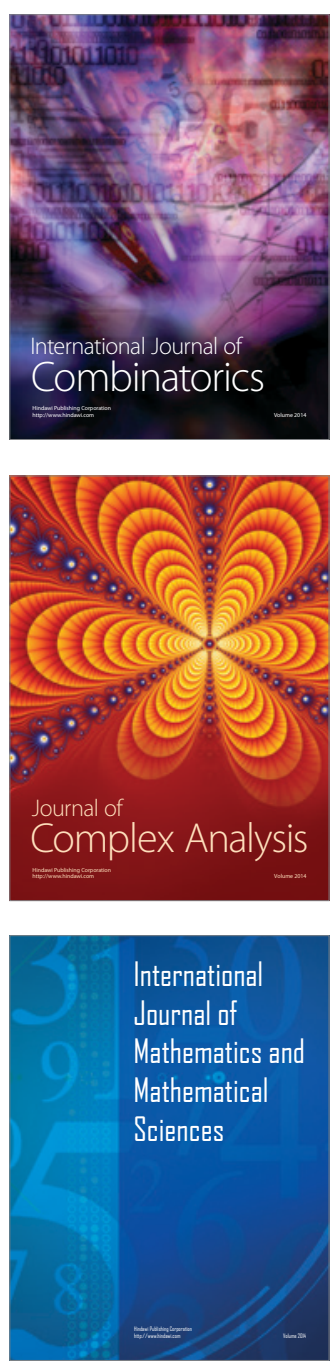
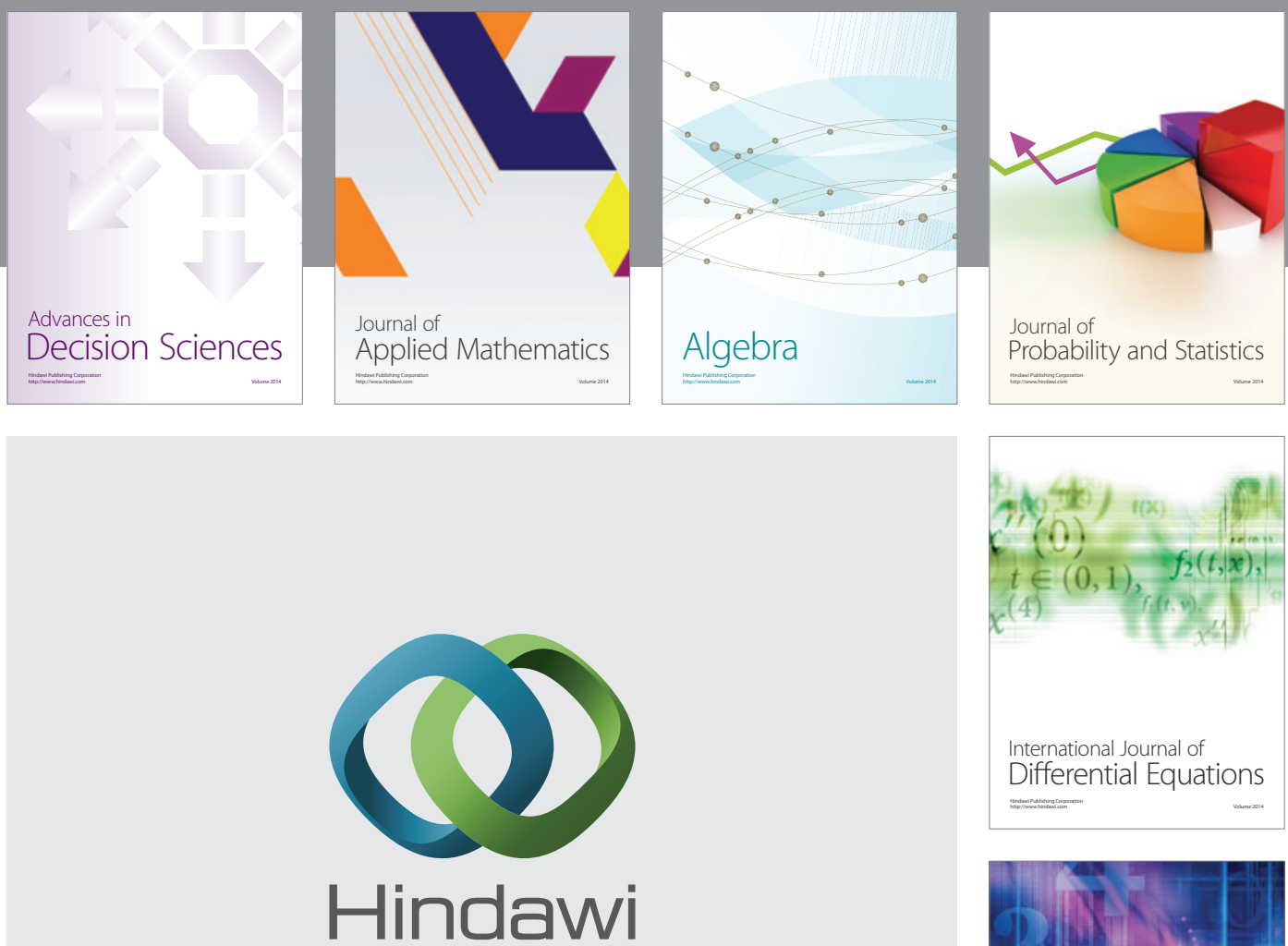

Submit your manuscripts at http://www.hindawi.com
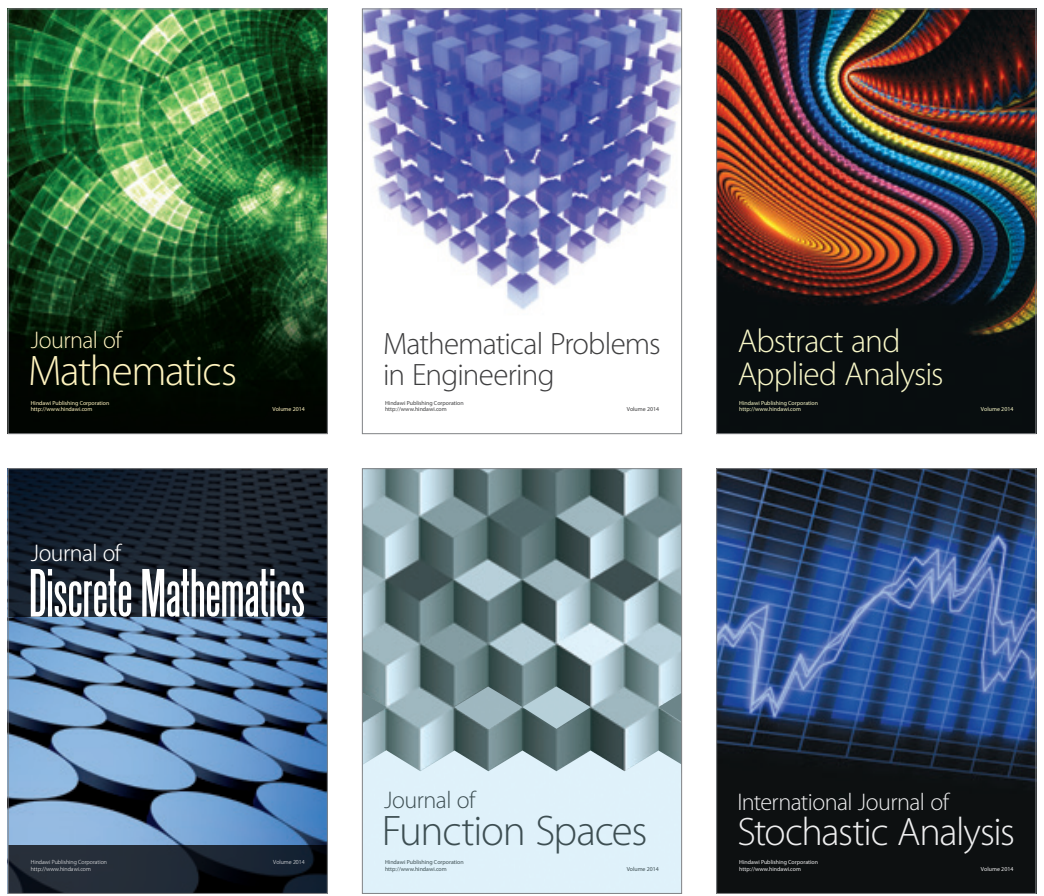

Journal of

Function Spaces

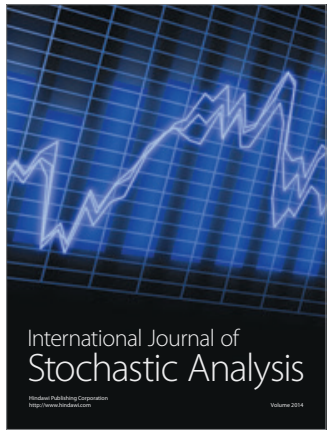

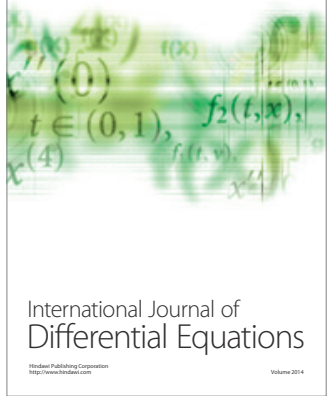
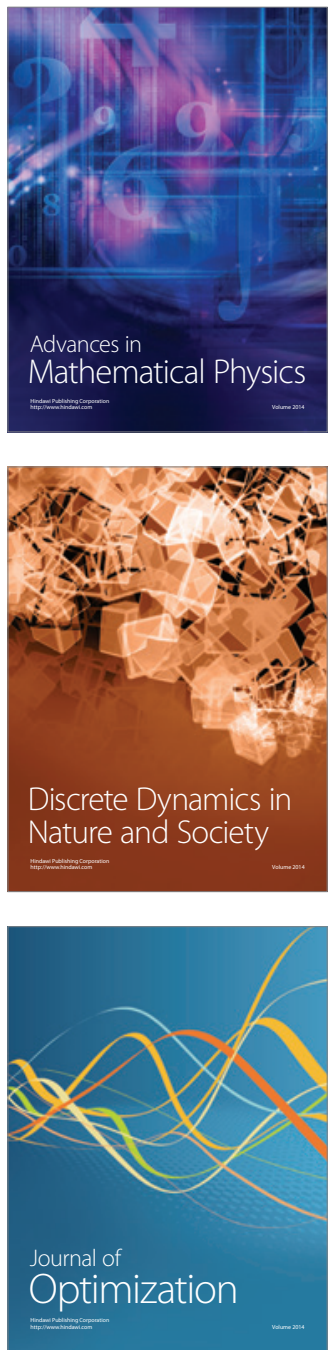